\title{
FIBER SEGMENTATION IN CRACK REGIONS OF STEEL FIBER REINFORCED CONCRETE USING PRINCIPAL CURVATURE
}

\author{
MARKUS KRONENBERGER ${ }^{\bowtie, 1,2}$, KATJA SchladitZ ${ }^{1}$, BERnd HAMANN ${ }^{3}$ AND \\ HANS HAGEN ${ }^{2}$ \\ ${ }^{1}$ Image Processing Group, Fraunhofer ITWM, 67663 Kaiserslautern, Germany; ${ }^{2}$ Computer Graphics and HCI \\ Group, University of Kaiserslautern, 67663 Kaiserslautern, Germany; ${ }^{3}$ Department of Computer Science, \\ University of California, Davis, CA 95616, U.S.A. \\ e-mail: markus.kronenberger@itwm.fraunhofer.de, katja.schladitz@itwm.fraunhofer.de, \\ hamann@cs.ucdavis.edu, hagen@cs.uni-kl.de \\ (Received February 22, 2018; revised May 5, 2018; accepted May 10, 2018)
}

\begin{abstract}
This paper tackles the non-trivial image-processing task to segment hook-ended fibers in three-dimensional images. For this purpose, a novel segmentation method is presented that relies on the following observation: For a single fiber the configurations of principal curvatures that can occur on its surface are limited. Deviations from these configurations indicate potential overlaps of fibers. The method that was developed based on this observation is used to separate several simulated clusters of touching fibers as a proof-of-concept. Further, it is applied to two images of cracked steel fiber reinforced concrete specimens arising from a 4-point bending test. The method's performance is compared to manual separation. Overall, we can state that the proposed method yields satisfying results when data meets the following criteria: Low fiber volume density, circular fiber cross section and sufficient spatial resolution of fiber-fiber contacts.
\end{abstract}

Keywords: fiber reinforced concrete, fiber segmentation, image analysis, X-ray micro-computed tomography.

\section{INTRODUCTION}

In civil engineering, fiber reinforced materials enjoy growing popularity among scientists and engineers. One important example is steel fiber reinforced concrete (SFRC). This is a composition of concrete and steel fibers that are added to the cement matrix in liquid state. In contrast to plain concrete the resulting material is able to bear higher tensile stresses after crack formation (Thomas and Ramaswamy, 2007). Application examples are commercial buildings, industrial floors or tunnel linings. In all of these applications, the post-crack behavior is of particular interest. After a failure of the concrete, the interplay between fibers and matrix is essential for counteracting a further crack propagation. For an in-depth review of the mechanics of crack formation and propagation the reader is referred to Afroughsabet et al. (2016).

Conventional approaches for investigating cracked SFRC require the destruction of the crack region. In contrast, X-ray micro-computed tomography $(\mu \mathrm{CT})$ allows to explore the material non-destructively based on three-dimensional (3D) images. This technique was used, e.g., by Schnell et al. (2011) in order to quantitatively analyze cracked SFRC. They showed that the alignment of fibers with respect to the crack has a higher influence on the tensile stress that the material can bear than the fiber volume fraction. This demonstration was possible by a global analysis using generalized projection lengths obtained as a by-product when measuring the integral of mean curvature (Ohser and Schladitz, 2009). This analysis interprets the fiber system as a stationary random closed set and thus does require only a separation of the fiber system (foreground) from the concrete (background).

The aforementioned investigation was possible without a segmentation of individual fibers. However, such a pre-processing would allow to analyze each fiber separately. As a result, a variety of geometric characteristics could be obtained directly. See Vecchio et al. (2012) for object features. These, in turn, describe the fiber geometry and therefore can be used to quantify the deformation that a fiber experienced during crack formation. Moreover, it would also be possible to determine the contribution of each fiber to the load transmission. Even heightdependent investigations of the crack region would be conceivable.

In the literature concerning fiber reinforced composites, a wide range of methods have been proposed in order to handle a segmentation of individual fibers in 3D images. Basically, these methods either perform a reasonable oversegmentation and afterwards trace single fibers or 
they directly separate fibers at their contact areas. Here, over-segmentation denotes a division into more segments than intended.

A number of researchers have developed methods for almost straight unidirectional (Requena et al., 2009; Latil et al., 2011; Czabaj et al., 2014; Emerson et al., 2017) or multidirectional fibers (Sencu et al., 2016). All of them have in common that they first detect center points of fibers in 2D slices and afterwards trace them throughout the 3D image.

Herrmann et al. (2016) compute local orientation vectors using the Hessian matrix and cluster them in order to determine the preferred orientation of overlapping fibers. Clusters consisting of uniquely oriented and completely straight fibers could simply be separated based on these orientations.

Apart from that, Altendorf (2011) combines radius, orientation and probability measurements in order to determine center points away from locations where fibers are crossing. Afterwards center line pieces are traced and simultaneously dilated using the estimated fiber radius. The result are reconstructed fiber parts that are disconnected at crossing locations. Finally, angle differences between the orientation vectors at the ends of fiber parts and the connection vectors between the ends are compared in order to unite the best matching parts.

The separation of curved fibers is also subject of current research. Gaiselmann et al. (2013), for example, assume fibers to have a previously known radius and an approximately constant circular cross section. By combining these two assumptions with the Euclidean distance transformation measuring the distance to the fiber surface they extract center lines by a radius-dependent thresholding. However, when this approach is applied to CT images, an over-segmentation of such lines can occur due to discretization effects and noise. Therefore, center line pieces are traced in a subsequent step. Randomly pieces are joined that fulfill user selected distance and angle criteria. Afterwards, the candidate with the highest mean value, computed over an enlarged overlapping subset of the initial CT image, is considered to be a true fiber. This procedure is repeated until all fiber pieces are processed.

Pinter et al. (2016) use the same assumptions as the method described before, but suggest to use a circular voting filter, which they define as weighted geometric mean of two measurements: The first part is a surface normal overlap measure, which exploits that for a fixed radius and a continuously circular cross section one can find a high response close to the center line. The second part is a coherence measure that considers the eigenvalues of the structure tensor to decide if a structure is cylindrically shaped. This shape-related interpretation of the eigenvalues goes back to Frangi et al. (1998). Center lines can then be obtained by a global thresholding that is controlled by the user. In this case, too, an over-segmentation can occur when the method is applied to CT images. Local orientation information are used to perform a suitable reconnection.

Teßmann et al. (2010), on the other hand, use the approach by Frangi et al. (1998) directly to identify center points. Afterwards, they use local orientation vectors, which are a by-product of the applied approach in order to trace the points to complete center lines.

Another popular concept for single fiber segmentation is template matching (Eberhardt and Clarke, 2002; Martin-Herrero and Germain, 2007; Weber et al., 2012). A 3D image is processed by comparing its local structure with a pre-defined template (line, ellipse, cylinder). The consensus between the local structure and the template provides information about the most probable positions of the center line of fibers as well as their orientations. Both information are exploited later to trace individual fibers.

In other applications, researchers had not only to deal with curved, but also deformed fibers. In order to tackle this complicated issue, Lux (2013) proposed an approach that first computes a skeleton and disassembles it to meaningful parts. Afterwards, the parts are properly assembled to complete fibers, which is controlled by several parameters. In order to make this approach more user-friendly, Huang et al. (2016) reduced the number of required parameters using the idea of punishing terms.

Up to now, the only method that directly segments fibers without the need of a subsequent tracing step was proposed by Viguié et al. (2013). This approach is able to separate curved and deformed fibers that slightly overlap. For this purpose, it estimates the orientation field inside of the fiber component using the main axis of inertia like in the case of Altendorf (2011). Afterwards, the method interprets high values of the gradient of this field as potential locations where fibers are overlapping and removes them.

All described approaches face problems when they are applied to overlapping fibers with hooked ends. Fig. 2 shows renderings of such fibers contained in cracked SFRC specimens. Here, we have to deal with straight, almost parallel fibers as well as strongly curved ends. For that reason, methods geared to almost straight fibers are not practical. However, also the 
other mentioned approaches do not lead to the desired result. All approaches, except the one by Viguié et al. (2013) partially or completely rely on local orientation information while tracing fibers. While this does not pose a problem when having fibers that are bent on a large scale, it can lead to problems when considering fibers with strong local bends like the hooked fiber ends in the present case. See Fig. 6g for an example. In such a situation orientation information can be misleading when tracing fibers.

On the contrary, the method suggested by Viguié et al. (2013) does not require any of the mentioned assumptions and is capable to perform the fiber separation without subsequent tracing step. Unfortunately, it is possible that fibers in SFRC are almost parallel and do overlap in the 3D image (Fig. 2). For parallel overlapping fibers the gradient of the orientation field is low as the orientations are similar or even identical. Therefore, the method is not able to separate fibers in such situations satisfactorily.

Thus, there is a need for a method that can separate fibers with the characteristics that can be found in cracked SFRC. This paper contributes by

- giving a thorough overview over existing approaches for single fiber segmentation and

- presenting a novel segmentation method for hookended fibers in 3D images that can be controlled using only four input parameters.

\section{MATERIALS AND METHODS}

\section{CRACKED SFRC}

The cracked SFRC specimens that are considered within this work arise from a 4-point bending test. The Institute of Construction Material Technology manufactured a large beam of SFRC in cooperation

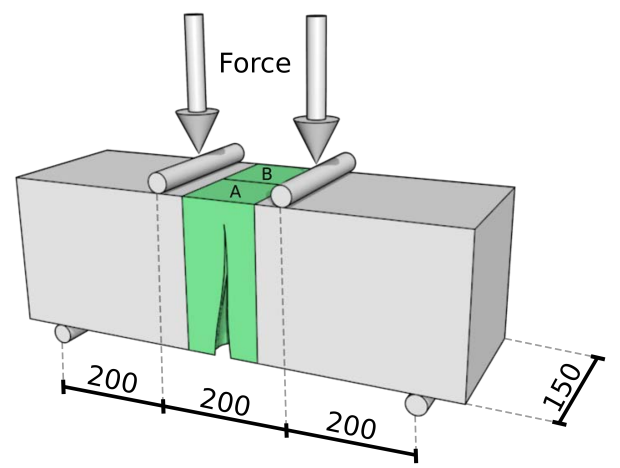

Fig. 1. Sketch of a 4-point bending test. Dimensions are given in millimetres. with a local ready-mixed concrete plant. The exact composition of the concrete (cement, aggregates, etc.) can be found in Bund (2011). For the reinforcement of the concrete steel fibers of type Dramix RC-80/60-BN with a diameter of $0.75 \mathrm{~mm}$ and an overall length of $60 \mathrm{~mm}$ were added. The mixing of both components was performed in liquid state at a dosage of $25 \mathrm{~kg}$ of steel fibers per cubic meter concrete. See Bund (2011) for further details. The prepared beam is positioned on two supporting pins, while two loading pins are used in order to apply force to the beam. See Fig. 1 for a sketch of the test setup. After crack formation, the interesting part shown in green is cropped out and cut in two halves, $\mathrm{A}$ and $\mathrm{B}$, in order to get a better aspect ratio for the subsequent $\mathrm{CT}$ scan.

3D images of specimens $\mathrm{A}$ and $\mathrm{B}$ are acquired using $\mu C T$. See the volume renderings in Fig. 2. They have a size of $737 \times 573 \times 1510$ and $746 \times 559 \times 1530$ voxel. This approximately corresponds to $6.9 \times 5.4 \times$ 14.2 and $7.03 \times 5.27 \times 14.42 \mathrm{~cm}^{3}$ for an isotropic voxel side length of $94.27 \mu \mathrm{m}$.

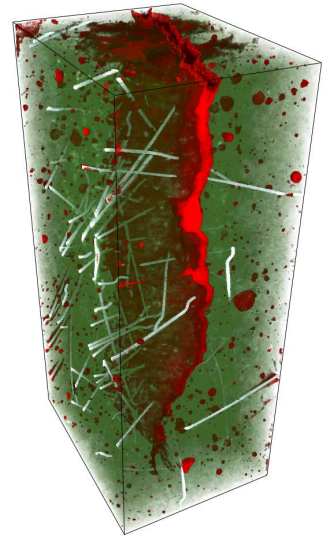

(a)

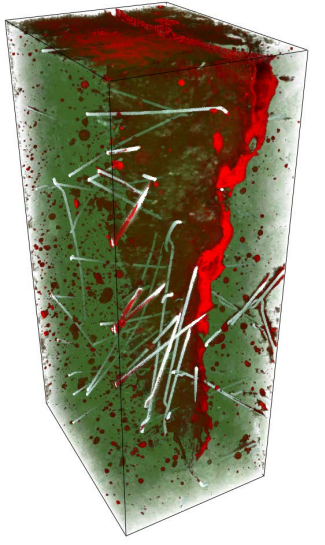

(b)
Fig. 2. 3D renderings of two cracked SFRC specimens. Concrete mass is indicated in green, crack in red, and fibers in white color. Specimens: Institute of Construction Material Technology, University of Kaiserslautern. Imaging: Fraunhofer ITWM.

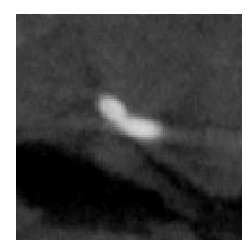

(a)

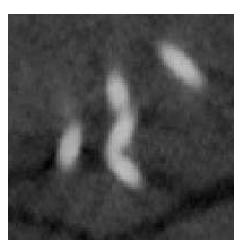

(b)
Fig. 3. Blurred contacts of two (a) or three (b) fibers in the $3 D$ image of specimen $A$. 
The reinforcing fibers are made of steel and thus do not physically overlap. However, in the 3D image, virtual overlap does occur (Fig. 3), mainly due to the so-called partial volume effect (PVE): Computed tomography is based on the discrete projection images that the flat bed detector generates in each angular position of the specimen. Thus, each voxel represents a small subvolume of the specimen. Voxels at the boundary of two material components carry a weighted mean of the gray values corresponding to each of the two components. Thus, the boundaries between components of strongly differing X-ray absorption contrasts are blurred. Clearly, this effect can be reduced by increasing the resolution. However, this measure is limited by the requirement to image a representative volume.

In the present case, the experimental setup dictates the size and shape of the two specimens. In order to obtain meaningful results, the complete crack and all crossing fibers have to be covered. This is favorable for the subsequent evaluation, but the corresponding specimens are quite large. While $\mu \mathrm{CT}$ devices allow for spatial resolutions down to the submicrometer range, the achievable resolution always depends on the size of the specimen which is to be imaged. Thus, the obtained voxel side length of $94.27 \mu \mathrm{m}$ has to be considered as trade-off in order to handle the large size of the specimens. In addition, the prismatic shape can lead to problems when acquiring 3D images. Then X-rays have to pass through significantly different amounts of material. This can cause various CT artifacts. In the case of specimens A and B slight cupping artifacts

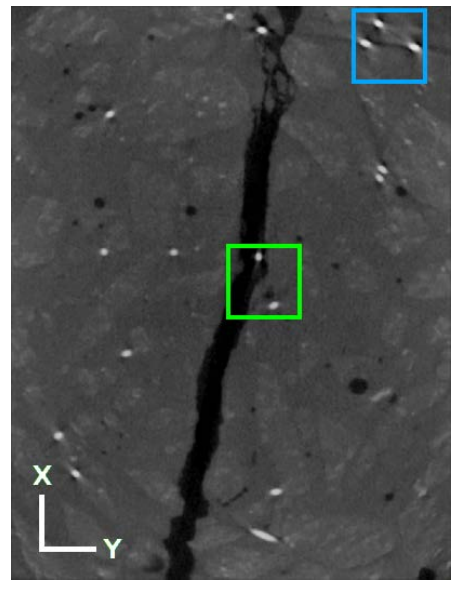

(a)

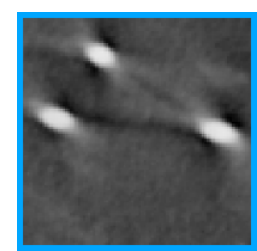

(b)

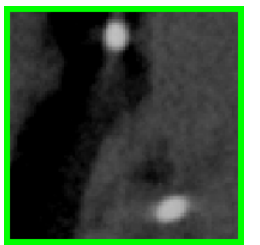

(c)
Fig. 4. Gray-value distribution in an xy-slice of the $3 D$ image of specimen $A$. The cross sections of fibers close to image corners show pronounced disturbances by streaks due to beam hardening artifacts $(b)$ compared to their equivalents in the middle of the image (c). in the concrete matrix are notable. Further, effects similar to beam hardening within the included fibers emerge. Here, the cupping occurs along the structure and differs with alignment to the X-ray direction. The result are gray-value fluctuations within the fibers and streak effects around their surface. These cause a change of the otherwise heterogeneous fiber cross section shape (Fig. 4). Moreover, they worsen the blurring effect between different fibers. For a more detailed description of such physics-based X-ray CT artifacts the reader is referred to Buzug (2008).

PVE as well as CT artifacts influence how exact the steel fibers can be captured by the acquired 3D images. While artifacts can be reduced, the PVE is $\mu \mathrm{CT}$ inherent and thus determines the lower bound of how fine grained the fibers can be captured. Due to the PVE at least one layer of voxels can not be unambiguously assigned to steel fibers or concrete. As a consequence, an inaccuracy of one voxel (around $0.09 \mathrm{~mm}$ ) at the interface between the materials needs to be taken into consideration when evaluating the fibers. Note that the PVE can be reduced by increasing the resolution. However, this is not practicable in the present case due to the trade-off between specimen size and resolution.

\section{SINGLE FIBER SEGMENTATION METHOD}

Our method for segmenting single fibers is based on the analysis of principal curvatures on the surface of fibers. In the following, we first repeat the corresponding mathematical background and afterwards we explain how we use the concept within the proposed method.

\section{Principal Curvatures}

Principal curvatures are a known concept from differential geometry that is useful for describing the shape of surfaces locally. For the subsequent explanation of the proposed segmentation method we make use of the definitions by do Carmo (1976) and Kreyszig (1991).

Using osculating circles: Let us consider a point $p$ on a regular plane curve. Then, a circle can be found that optimally approximates the curve infinitesimally close to $p$. This circle is called osculating circle. Using its radius $r$, the curvature at $p$ is defined as:

$$
\kappa=\frac{1}{r}
$$

In the surface case this idea is applied too. There, for any point one can extract a plane curve by intersecting 


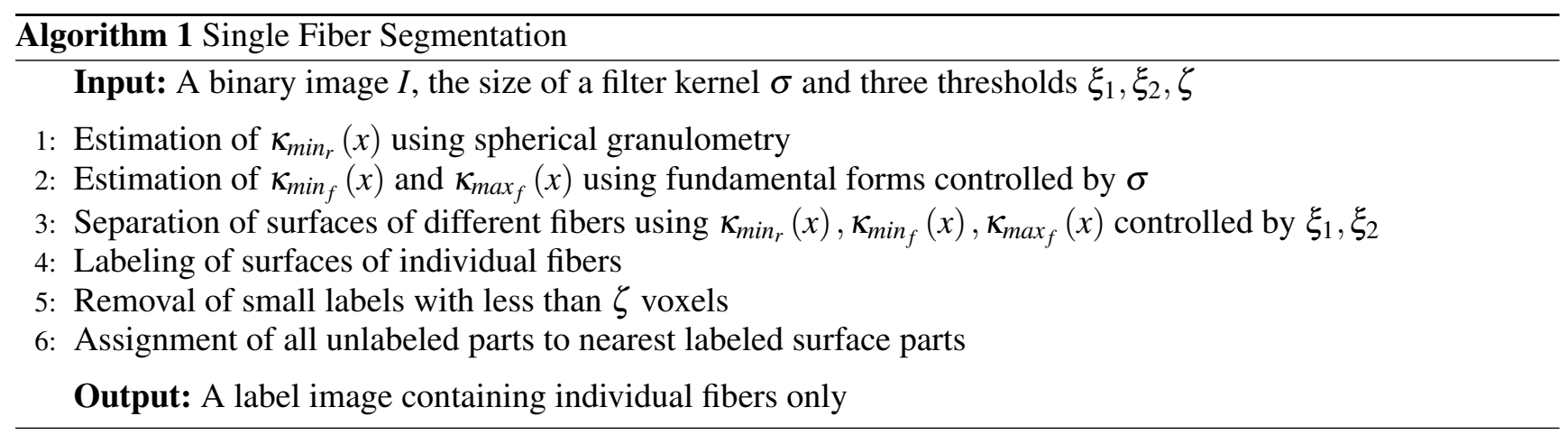

the surface with a plane, described by the normal vector and a vector lying within the tangent plane. By running through all possible tangent vectors and computing the curvatures of the resulting plane curves, one maximum $\kappa_{\max _{r}}$ and one minimum $\kappa_{\min _{r}}$ can be identified, which are called principal curvatures.

Using fundamental forms: Let $S$ be a differentiable surface in $3 \mathrm{D}$ that is defined by an explicit function $f: \mathbb{R}^{2} \rightarrow \mathbb{R}^{3}$. This function assigns every pair $(u, v) \in \mathbb{R}^{2}$ to a point of $S \subset \mathbb{R}^{3}$. $f_{i}$ with $i \in\{u, v\}$ denote the first partial derivatives w.r.t. $i$ and $f_{i j}$ with $i, j \in\{u, v\}$ the partial derivatives of second order of $f$. Then, at each point of $S$, the normal vector is given by

$$
N=\frac{\left[f_{u}, f_{v}\right]}{\left\|\left[f_{u}, f_{v}\right]\right\|_{2}},
$$

where $[\cdot, \cdot]$ denotes the cross product. Using these notations we define the first and second fundamental form as

$$
\begin{gathered}
\mathscr{F}_{1}=\left(\begin{array}{cc}
\left\langle f_{u}, f_{u}\right\rangle & \left\langle f_{u}, f_{v}\right\rangle \\
\left\langle f_{v}, f_{u}\right\rangle & \left\langle f_{v}, f_{v}\right\rangle
\end{array}\right), \\
\mathscr{F}_{2}=\left(\begin{array}{ll}
\left\langle f_{u u}, N\right\rangle & \left\langle f_{u v}, N\right\rangle \\
\left\langle f_{v u}, N\right\rangle & \left\langle f_{v v}, N\right\rangle
\end{array}\right),
\end{gathered}
$$

where $\langle\cdot, \cdot\rangle$ denotes the scalar product. In simple terms $\mathscr{F}_{1}$ allows to calculate lengths of curves or areas of regions on deformed surfaces. $\mathscr{F}_{2}$, on the other hand, is an operator that describes the deformation of a surface by measuring the changing of $N$. The principal curvatures $\kappa_{\min }$ and $\kappa_{\max _{f}}$ can then be defined using $\mathscr{F}_{1}$ and $\mathscr{F}_{2}$ as

$$
\begin{aligned}
\kappa_{\text {min }} & =\mathscr{H}-\sqrt{\mathscr{H}^{2}-\mathscr{K}}, \\
\kappa_{\text {max }_{f}} & =\mathscr{H}+\sqrt{\mathscr{H}^{2}-\mathscr{K}},
\end{aligned}
$$

with

$$
\mathscr{H}=\frac{1}{2} \operatorname{trace}\left(\frac{\mathscr{F}_{2}}{\mathscr{F}_{1}}\right), \quad \mathscr{K}=\frac{\operatorname{det}\left(\mathscr{F}_{2}\right)}{\operatorname{det}\left(\mathscr{F}_{1}\right)} .
$$

\section{Proposed algorithm}

The input for our method is a binary 3D image $I: \Omega \rightarrow\{0,1\}$, where $\Omega$ is a cuboidal subset of $\mathbb{Z}^{3}$. Let 1 indicate the fiber component $X \subset \Omega$ and let 0 indicate the background $\bar{X}=\Omega \backslash X$. We will call $x \in \Omega$ a voxel for the rest of this work. Further, we consider the 26-adjacency system as neighborhood of a voxel, i.e., $x$ is a neighbor of $y$ iff $\|x-y\|_{\infty} \leq 1$ with $y \in \Omega$. Here, $\|\cdot\|_{\infty}$ denotes the maximum norm.

Algorithm 1 presents a high-level description of the suggested single fiber separation method. Next, we are going to explain each of its steps in detail.

Step 1 - Minimum principal curvature via granulometry: In the first step of the proposed algorithm the spherical granulometry distribution $g(x)$ of $I$ is computed (Soille, 2013). $g(x)$ gives the radius ${ }^{1}$ of the largest ball that covers $x$ and is completely contained in $X$. For all voxel $x$ of the background $\bar{X}$ the function $g(x)$ returns zero. See Soille (2013) for details how to efficiently implement such a measure using morphological opening.

Unfortunately, noise and discretization artifacts are captured close to the surface. For this reason, the following adaptation is applied: We use the internal gradient (Rivest et al., 1992) with a $3 \times 3 \times 3$ cube as structuring element in order to identify the set $X_{S}$ of all voxels representing the surface of the fiber component $X$. Afterwards we replace their values with the values of the nearest neighbors in $X \backslash X_{s}$ using the Euclidean distance $d(\cdot, \cdot)$ :

$$
g^{\prime}(x)= \begin{cases}g\left(\underset{y \in X \backslash X_{S}}{\operatorname{argmin}}\{d(x, y)\}\right), & \text { if } x \in X_{S} \\ g(x), & \text { otherwise. }\end{cases}
$$

It is possible that several voxels $y$ have the same minimal distance $d(x, y)$ in Eq. 3 and therefore argmin does not have to be unique. We solve this in our

\footnotetext{
${ }^{1}$ Usually, the granulometry computes the diameter that is twice the radius.
} 
BENT FIBER

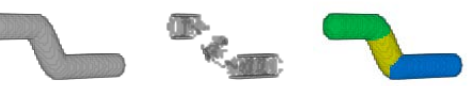

(a) $\xi_{1}=0.15, \xi_{2}=0.05$

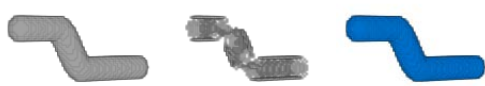

(b) $\xi_{1}=0.15, \xi_{2}=0.15$
FIBER WITH ELLIPTICAL CROSS SECTION
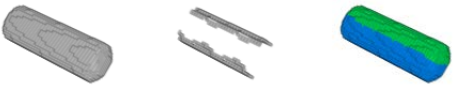

(c) $\xi_{1}=0.15, \xi_{2}=0.15$
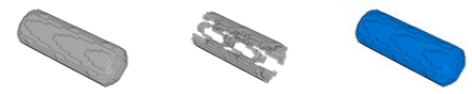

(d) $\xi_{1}=0.5, \xi_{2}=0.15$
OVERLAPPING FIBERS
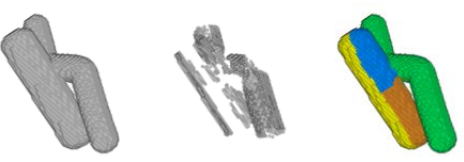

(e) $\xi_{1}=0.15, \xi_{2}=0.15$
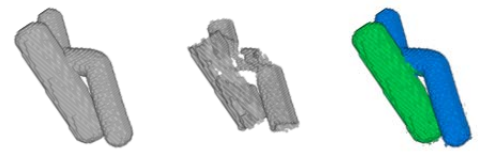

(f) $\xi_{1}=0.5, \xi_{2}=0.15$

Fig. 5. Parameter selection for different fiber shapes. In each case, the input for the proposed method, the separated surfaces, and the final result are visualized. The value of $\sigma$ was 2 , and the value of $\zeta 100$. Different colors indicate different connected components.

implementation by taking the first $y$ that fulfilled the condition.

Afterwards, an approximation for the minimum principal curvature is calculated using $g^{\prime}(x)$. For each voxel $x$ of the fiber surface $g^{\prime}(x)$ can be considered as estimation of the fiber radius. In the special case of a fiber, this radius extracted of a fitted ball comes close to the idea of the osculating circle and therefore we use $g^{\prime}(x)$ as radius $r$ in Eq. 1:

$$
\kappa_{\text {min }_{r}}(x)= \begin{cases}\frac{1}{g^{\prime}(x)}, & \text { if } x \in X_{s}, \\ 0, & \text { otherwise. }\end{cases}
$$

Please note that $\kappa_{\min _{r}}(x) \geq 0$ due to the fact that the radius estimated by the granulometry is also always larger than zero.

Step 2 - Principal curvatures via fundamental forms: In the second step the principal curvatures $\kappa_{\min _{f}}(x)$ and $\kappa_{\max _{f}}(x)$ based on the fundamental forms are estimated for $I$. The discretization of the fundamental forms to the discrete setting of binary images has already been described in previous work (Kronenberger et al., 2015). The subsequent computation of the principal curvatures, i.e., Eq. 2, is then straightforward. In contrast to the previous work we here switched the approach that we use for calculating normal vectors. Instead of using the center of mass approach like in Kronenberger et al. (2015), we here convolve 3D images with a Gauss filter of size $\sigma$ and afterwards use the idea of finite differences, as suggested, e.g., by Thirion and Gourdon (1995) or Wernersson et al. (2011) for deriving the needed normal vectors.
Step 3 - Surface separation: The surface of an almost straight fiber is described by a strongly negative minimum principal curvature $\left(\kappa_{\min } \ll 0\right)$ in combination with a maximum principal curvature close to zero $\left(\left|\kappa_{\max }\right| \approx 0\right)$. This is true except for fiber ends and for hooked parts where $\left|\kappa_{\max }\right|$ increases. Using this knowledge, we can identify overlapping areas by investigating the compliance of $\kappa_{\max _{f}}(x)$ and $\kappa_{\min _{f}}(x)$ with those assumptions. In our experiment, some adaptations turned out to be helpful:

First, pronounced gray-value fluctuations cause the radius of the discrete fibers to vary and thus it is complicated to determine a value range of $\kappa_{\min }$ only allowing for single fibers. For this reason, we add $\kappa_{\min _{r}}(x)$ to $\kappa_{\min _{f}}(x)$ and take the absolute value. This makes sense, as both measures nearly reflect the same quantity, but with different sign. Hence, the resulting term should be close to zero.

Second, the terms $\left|\kappa_{\text {min }_{f}}(x)+\kappa_{\text {min }_{r}}(x)\right|$ and $\left|\kappa_{\max }(x)\right|$ should be close to zero, when evaluated for the lateral surface of a single fiber. Since "close to zero" is always a relative statement, we scale both terms with $\kappa_{\min _{r}}(x)$ so that the terms can be interpreted more intuitively. $\kappa_{\min _{r}}(x)$ is defined using the idea of osculating circles and therefore illustrative.

Using the previously discussed aspects we came up with two conditions

$$
\begin{aligned}
\left|\kappa_{\text {min }_{f}}(x)+\kappa_{\text {min }_{r}}(x)\right| & <\xi_{1} \cdot \kappa_{\text {min }_{r}}(x), \\
\left|\kappa_{\max _{f}}(x)\right| & <\xi_{2} \cdot \kappa_{\text {min }_{r}}(x),
\end{aligned}
$$

to separate surfaces of different fibers by removing non-fiber-like parts: 

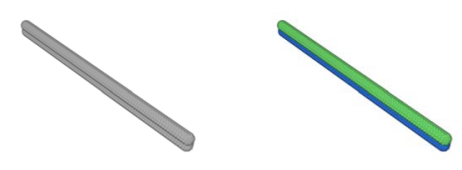

(a)

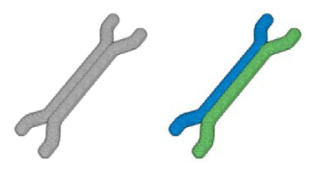

(d)

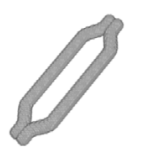

(e)

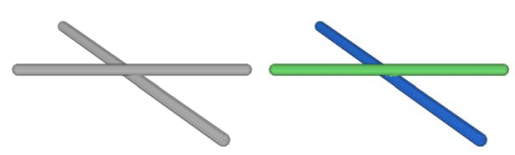

(b)

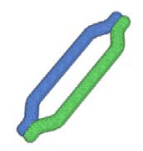

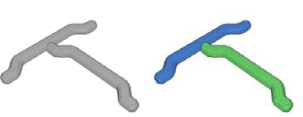

(f)
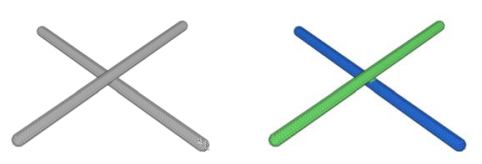

(c)

Fig. 6. Simulated fiber clusters and their separated versions obtained with our method. Different colors represent various connected components.

$$
\tilde{X}_{s}(x)= \begin{cases}1, & \text { if Eqs. } 4 \text { and } 5 \text { are satisfied } \\ 0, & \text { otherwise }\end{cases}
$$

Here, $\xi_{1}$ and $\xi_{2}$ control the separation process and can be used to compensate for bent fibers as well as small deformations in the cross sections. Their values should be selected between 0 and around 1 . Note that due to the nature of the granulometry and its adjustment in Step 1, the difference between the approximation $\kappa_{\min _{r}}(x)$ and the estimation $\kappa_{\min _{f}}(x)$ increases with deviation from the ideal fiber shape.

Step 4-Labeling: After removing surface voxels that connect different fibers in Step 3, we label the connected components (Soille, 2013) on $\tilde{X}_{s}(x)$ w.r.t. a 26-adjacency.

Step 5 - Clean up: Due to discretization and noise it is possible that in Step 4 some labels do not represent fiber surfaces and consist of comparably less voxels. That is why we remove all connected components with less than $\zeta$ voxels.

Step 6 - Voxel to label assignment: In the last step, we run through every voxel of the fiber component that is not yet labeled and search for its nearest neighbor that already has a label. Afterwards we assign the label found to the starting voxel.

\section{Parameter selection}

The proposed method is controlled by four parameters. The first parameter $\sigma$ is used to set the degree of smoothing that is applied within the principal curvature estimation using fundamental forms. It has to be selected according to the structure within the 3D image as well as the image quality. The reader is referred to the works of Thirion and Gourdon
(1995) as well as Wernersson et al. (2011) for details concerning a good choice of this parameter. In all of our experiments it was sufficient to select $\sigma$ as 2 .

The actual separation of the surfaces of different fibers is controlled by $\xi_{1}$ and $\xi_{2}$. Fig. 5 illustrates the effect of different choice of these two parameters. If the value for $\xi_{2}$ is chosen too small an oversegmentation along a bent fiber can occur (Fig. 5a). We can therefore think of $\xi_{2}$ as controlling the allowed bending of a fiber. A higher value has to be selected in order to achieve the desired result (Fig. 5b).

In cracked SFRC changes from a circular to an elliptic cross section are possible as, e.g., visible in Fig. 4. A suitable choice of $\xi_{1}$ can solve oversegmentations occurring due to such elliptical cross sections (Fig. 5c and 5d).

In the case of overlapping fibers, as shown in Fig. $5 \mathrm{e}$ and $5 \mathrm{f}$, the parameters $\xi_{1}$ and $\xi_{2}$ have to be selected according to the findings described before. However, one could now draw the conclusion that the parameter values should always be selected high, but this is not the case. It is rather a compromise between choosing $\xi_{1}$ and $\xi_{2}$ as high as possible, but not too high since then the fibers are not separated any more.

The last parameter $\zeta$ is a threshold for removing separated surfaces parts that are too small to be considered as real fibers. In all of our experiments we selected $\zeta$ as 100 voxel.

\section{RESULTS}

\section{PROOF-OF-CONCEPT}

Several configurations with overlapping fibers were simulated in order to perform a proof-of-concept. 
All configurations are shown in Fig. 6. We selected configurations being similar to those typically found in 3D images of cracked SFRC (Fig. 2). Since fibers are initially straight, except for their hooked ends, it is possible that straight parts touch each other, requiring us to handle such configurations. We simulated three pairs of straight fibers with a diameter of eight voxels with overlapping parts crossing at angles $0^{\circ}, 45^{\circ}$ and $90^{\circ}$.

Configurations involving hooked ends are of special interest due to their more complicated geometry. Thus, we simulated several relevant cases, shown in the second row of Fig. 6.

Our method can separate all simulated clusters illustrated in Fig. 6. For all eight fiber pairs we set the value of the smoothing parameter $\sigma$ to 2 . The parameter values of $\xi_{1}$ and $\xi_{2}$, controlling the separation step, were set to 0.3 and 0.1 , respectively. The threshold value of $\zeta$ was set to 100 .

\section{APPLICATION}

We used the proposed method to separate fiber clusters in two 3D images of cracked SFRC, shown in Fig. 2. In order to apply the single fiber segmentation approach to these gray-scale images we performed the following pre-processing: First, a morphological closure filter was employed, using a ball with an approximate diameter of 2.31 voxels, to act as a structuring element. This step removed small variations in gray values, while avoiding an excessive blurring between different fibers.

For the further processing a segmentation of the fiber component in both images is necessary. This is done by a binarization using global gray-value

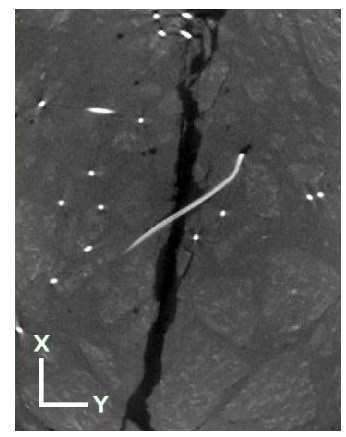

(a)

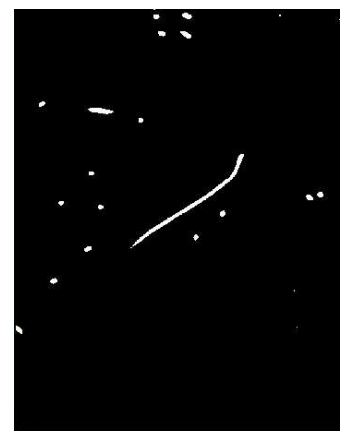

(b)
Fig. 7. Example binarization result of global thresholding (b) for one xy-slice of the $3 D$ image of specimen $A(a)$. Here, white color indicates the segmented fiber component and black color the background. thresholding (Fig. 7). For every voxel it is decided whether it belongs to the fiber component or not depending on a threshold $t$. This makes sense, as usually the gray-values representing steel have a higher value than the ones representing air or concrete. The value of $t$ was chosen high enough to remove blurring, but low enough to keep fibers or parts of them. A high value of $t$ had to be selected in order to reduce pronounced blurring effects between fibers in regions very close to image corners, see Fig. $8 \mathrm{a}$ to $8 \mathrm{c}$. At the same time, the value of $t$ must not be too high as this can cause fibers to disappear, as can be seen in Fig. 8d to $8 \mathrm{f}$. For the image of specimen A we set $t$ to 15750 , and for the image of specimen B we set it to 21000 .

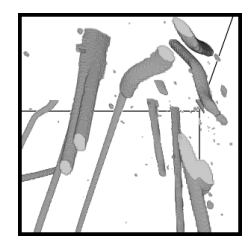

(a) $t=18000$

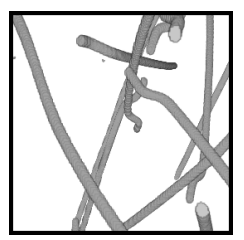

(d) $t=18000$

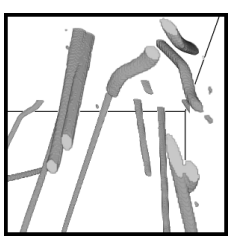

(b) $t=21000$

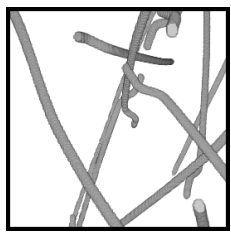

(e) $t=21000$

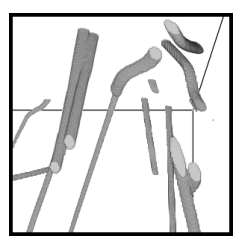

(c) $t=24000$

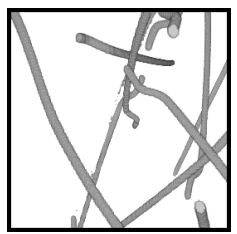

(f) $t=24000$
Fig. 8. Binarized fibers for specimen B using different threshold values.

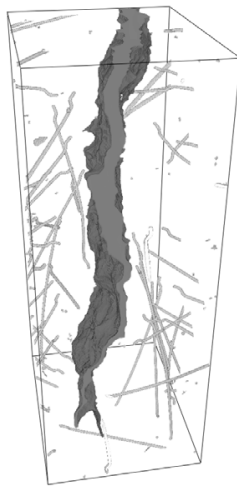

(a)

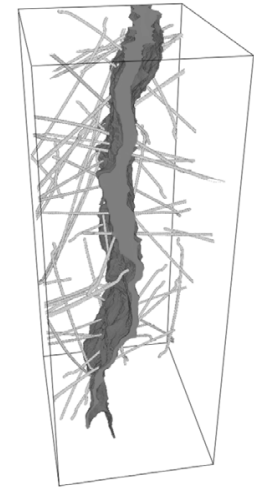

(b)
Fig. 9. Fiber of interest selection. $3 D$ renderings of the fiber component (indicated in light gray color) and the segmented crack (indicated in dark gray color) of specimen A. (a) shows all fibers and clusters that do not overlap with the crack and therefore will no longer be considered. Whereas (b) presents all fibers and clusters, which are separated into individual fibers in this experiment. 


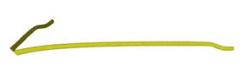

(a) $n=2$

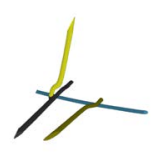

(f) $n=4$

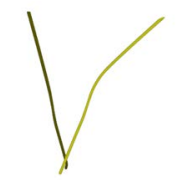

(b) $n=2$

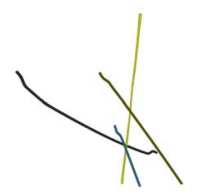

(g) $n=4$ (c) $n=2$

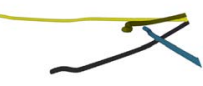

(h) $n=4$

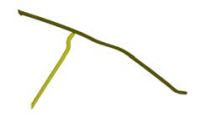

(d) $n=2$

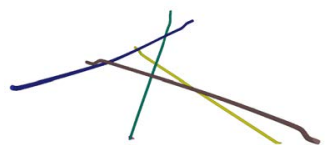

(i) $n=5$

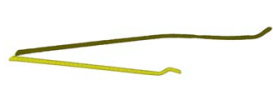

(e) $n=2$

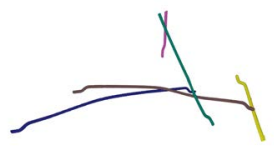

(j) $n=5$

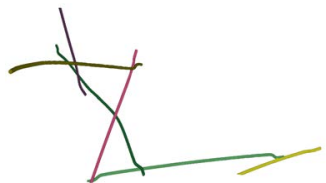

(k) $n=6$

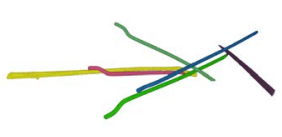

(1) $n=6$

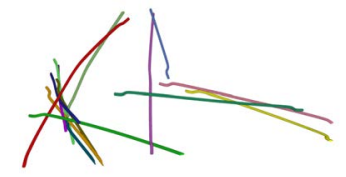

(m) $n=14$

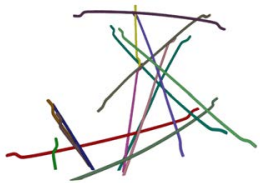

(n) $n=15$

Fig. 10. Single fiber segmentation of all clusters contained in the $3 D$ images of specimens $A$ and $B$ touching the segmented crack. Individual fibers are shown in different colors, with $n$ being the number of fibers.

Thresholding did segment fibers, in addition to segmenting very small objects due to noise. Therefore, we removed all objects consisting of less than 132 voxels. This choice seemed reasonable, as it is roughly the volume of a voxelized half-ball with a diameter of 7.96 voxels, corresponding to the diameter known from the production process of the fibers.

For the analysis of cracked SFRC only fibers crossing or touching the crack are of interest. We extracted the crack using global thresholding, manually removed sticking-out parts, and filled holes produced by the fibers using a closure filter with a ball of diameter 45.61 voxels as structuring element. Afterwards, we identified all fibers and clusters touching the crack via simple superimposition (Fig. 9).

The pre-processing steps produce two binary images only containing fibers and fiber clusters touching the segmented crack. We applied our single fiber segmentation method to both images. Again, we set $\sigma$ to 2. Due to bent fibers and elliptical cross sections we had to increase the values of $\xi_{1}$ and $\xi_{2}$ compared to the proof-of-concept case. For the binary image of specimen B we defined the values of $\xi_{1}$ as 0.4 and of $\xi_{2}$ as 0.2 . However, the change of the cross section was even more pronounced in the binary image of specimen A. As a consequence, we had to increase the value of $\xi_{1}$ to 0.45 . The value of $\zeta$ was 100 voxels in both cases.

The pre-processed 3D images of specimens A and $\mathrm{B}$ included overall 47 single fibers and 14 fiber clusters. Our method was able to separate all clusters properly, while leaving single fibers unchanged. Small clusters of 2 to 4 fibers as well as large clusters of 14 to 15 fibers had to be handled, see Fig. 10 showing segmentation results.

In order to validate our results, we visually compared them with a manual segmentation. We observed that the last step of our method - assignment of all unlabeled voxels to the nearest labels - does not always lead to a desirable or meaningful separation, see Fig. 11. In Figs. 11a and 11b the cross section of the fiber is not circular, being one assumption of our method; therefore, inaccuracies can result. However, such effects can also occur, less pronounced, in locations where the cross sections are circular, see Fig. 11c. In our targeted application - computing features of the fibers - such small inaccuracies of the fiber surface are statistically not important.

We performed our experiment on a computer running the Red Hat Enterprise Linux Workstation

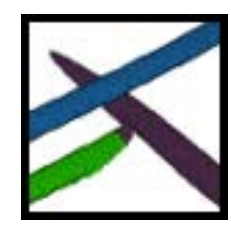

(a)

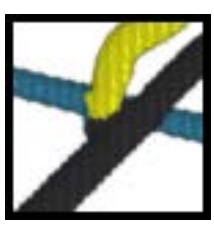

(b)

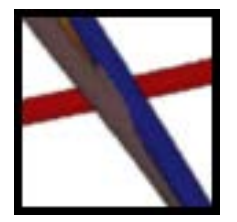

(c)
Fig. 11. Possible inaccurate segmentations.

Release 7.3 (64-bit), equipped with an Intel Core i7-6700 CPU and 64GB of memory. Starting with 
binary images of segmented fibers, our method had a runtime of around 47 minutes for specimen $\mathrm{A}$ and around 55 minutes for specimen $\mathrm{B}$. The main reason for these relatively long runtimes is our current naive implementation. Curvature estimation and adaptation of granulometry in the surface can be accelerated substantially via parallelized computations. However, our goal was to demonstrate the viability of our general algorithmic approach, and we will improve runtime behavior in the future.

\section{CONCLUSION}

A novel segmentation method for hook-ended fibers has been presented. Its performance has been demonstrated using simulated fiber clusters as a proofof-concept. Further, the method has been applied to fibers within 3D images of two cracked SFRC specimens arising from a 4-point bending test. In both cases it produced satisfying results even in the presence of parallel fibers, hooked ends and slightly deformed cross sections.

The suggested method is applicable when the following criteria are met:

Low fiber volume density: The fiber separation is realized by removing non-fiber-like surface parts. If too many fiber-fiber contacts are very close together the remaining surface parts in the overlap region can completely disappear. The resulting over-segmentation would then have to be solved manually.

Almost circular fiber cross section: Our experiments show that the suggested method is able to handle slightly deformed cross sections. Nevertheless, the underlying idea builds on the homogeneity of the principal curvatures for a cylindrical shape. Deviations from the circular cross section violate this assumption and therefore should be viewed with caution.

Sufficient spatial resolution of fiber-fiber contacts: The proposed segmentation method is based on local investigations of the surface shape. In order to be able to distinguish locally between a blurred overlap and a deformed fibre cross section the fiber-fiber contact has to appear at least slightly constricted. Otherwise the proposed method is not able to separate the fibers.

In the previous section, we have applied our method to 3D images of cracked SFRC specimens. It is planned to evaluate a complete series of such images in order to gain a deeper understanding of the postcrack behavior of such material. One advantage of the presented method is that the complete segmentation is controlled by four well interpretable parameters.

\section{ACKNOWLEDGEMENT}

The authors thank Frank Schuler and Bianca Bund for preparing the two SFRC specimens and fruitful discussions. This research has been supported by the German research foundation (DFG) within the IRTG 2057 "Physical Modeling for Virtual Manufacturing Systems and Processes" and the Fraunhofer High Performance Center Simulation and Software based Innovation.

\section{REFERENCES}

Afroughsabet V, Biolzi L, Ozbakkaloglu T (2016). Highperformance fiber-reinforced concrete: a review. J Mater Sci 51:6517-51.

Altendorf H (2011). 3D Morphological Analysis and Modeling of Random Fiber Networks. PhD Thesis. École Nationale Supérieure des Mines de Paris; Technische Universität Kaiserlautern.

Bund B (2011). Untersuchung des Nachrissverhaltens von Stahlfaserbeton mithilfe der Computer-Tomographie. Diplomarbeit. Technische Universität Kaiserslautern.

Buzug TM (2008). Computed Tomography: From Photon Statistics to Modern Cone-Beam CT. Berlin, Heidelberg: Springer.

Czabaj MW, Riccio ML, Whitacre WW (2014). Numerical reconstruction of graphite/epoxy composite microstructure based on sub-micron resolution $\mathrm{X}$ ray computed tomography. Compos Sci Technol 105:174-82.

do Carmo M (1976). Differential geometry of curves and surfaces. Englewood Cliffs: Prentice-Hall.

Eberhardt C, Clarke A (2002). Automated reconstruction of curvilinear fibres from 3D datasets acquired by X-ray microtomography. J Microsc 206:41-53.

Emerson MJ, Jespersen KM, Dahl AB, Conradsen K, Mikkelsen LP (2017). Individual fibre segmentation from 3D X-ray computed tomography for characterising the fibre orientation in unidirectional composite materials. Compos Part A Appl Sci 97:83-92.

Frangi AF, Niessen WJ, Vincken KL, Viergever MA (1998). Muliscale vessel enhancement filtering. In: Wells WM, Colchester A, Delp S, eds. Proc 1st Int Conf Med Image Computing and Computer-Assis Interven, MICCAI '98. Lect Not Comput Sci 1496:130-7.

Gaiselmann G, Manke I, Lehnert W, Schmidt V (2013). Extraction of curved fibers from 3D data. Image Anal Stereol 32:57-63.

Herrmann H, Pastorelli E, Kallonen A, Suuronen JP (2016). Methods for fibre orientation analysis of X-ray tomography images of steel fibre reinforced concrete (SFRC). J Mater Sci 51:3772-83. 
Huang X, Wen D, Zhao Y, Wang Q, Zhou W, Deng D (2016). Skeleton-based tracing of curved fibers from 3D X-ray microtomographic imaging. Results Phys 6:170-7.

Kreyszig E (1991). Differential geometry. Mineola: Dover.

Kronenberger M, Wirjadi O, Freitag J, Hagen H (2015). Gaussian curvature using fundamental forms for binary voxel data. Graph Models 82:123-36.

Latil P, Orgéas L, Geindreau C, Dumont P, Du Roscoat SR (2011). Towards the 3D in situ characterisation of deformation micro-mechanisms within a compressed bundle of fibres. Compos Sci Technol 71:480-8.

Lux J (2013). Automatic segmentation and structural characterization of low density fibreboards. Image Anal Stereol 32:13-25.

Martin-Herrero J, Germain C (2007). Microstructure reconstruction of fibrous c/c composites from X-ray microtomography. Carbon 45:1242-53.

Ohser J, Schladitz K (2009). 3D images of materials structures: Processing and analysis. Chichester: Wiley.

Pinter P, Bertram B, Weidenmann KA (2016). Novel method for the determination of fibre length distributions from $\mu \mathrm{CT}$-data. Proc Conf Indust Comput Tomo iCT.

Requena G, Fiedler G, Seiser B, Degischer P, Di Michiel M, Buslaps T (2009). 3D-quantification of the distribution of continuous fibres in unidirectionally reinforced composites. Compos Part A Appl Sci 40:152-63.

Rivest JF, Soille P, Beucher S (1992). Morphological gradients. Nonlinear Image Processing III SPIE/SPSE 1658:139.

Schnell J, Breit W, Schuler F (2011). Use of computertomography for the analysis of fibre reinforced concrete. In: Sruma V, ed. Proc fib Symp Prague 2011. 583-6.

Sencu R, Yang Z, Wang Y, Withers P, Rau C, Parson
A, Soutis C (2016). Generation of micro-scale finite element models from synchrotron X-ray CT images for multidirectional carbon fibre reinforced composites. Compos Part A Appl Sci 91:85-95.

Soille P (2013). Morphological image analysis: Principles and applications. Berlin, Heidelberg: Springer.

Teßmann M, Mohr S, Gayetskyy S, Haßler U, Hanke R, Greiner G (2010). Automatic determination of fiberlength distribution in composite material using 3D CT data. EURASIP J Adv Sign Proc 2010:545030.

Thirion JP, Gourdon A (1995). Computing the differential characteristics of isointensity surfaces. Comput Vision Image Und 61:190-202.

Thomas J, Ramaswamy A (2007). Mechanical properties of steel fiber-reinforced concrete. J Mater Civil Eng 19:385-92.

Vecchio I, Schladitz K, Godehardt M, Heneka M (2012). $3 \mathrm{D}$ geometric characterization of particles applied to technical cleanliness. Image Anal Stereol 31:163-74.

Viguié J, Latil P, Orgas L, Dumont P, du Roscoat SR, Bloch JF, Marulier C, Guiraud O (2013). Finding fibres and their contacts within 3D images of disordered fibrous media. Compos Sci Technol 89:202-10.

Weber B, Greenan G, Prohaska S, Baum D, Hege HC, Mller-Reichert T, Hyman AA, Verbavatz JM (2012). Automated tracing of microtubules in electron tomograms of plastic embedded samples of Caenorhabditis elegans embryos. J Struct Biol 178:129-38.

Wernersson ELG, Hendriks CLL, Brun A (2011). Accurate estimation of gaussian and mean curvature in volumetric images. In: Proc 2011 Int Conf 3D Imaging Modeling Process Visual Transmis. 312-7. 